\title{
MASSOTHÉRAPIE AVEC BALLES : HISTOIRE ET TECHNIQUE D'APPLICATION
}

\section{ARTICLE D'EXAMEN}

BESSA, Vicente Alberto Lima ${ }^{1}$

BESSA, Maria Fátima de Sousa ${ }^{2}$

BESSA, Vicente Alberto Lima. BESSA, Maria Fátima de Sousa. Massothérapie avec balles : histoire et technique d'application. Revista Científica Multidisciplinar Núcleo do Conhecimento. 04 année, Ed. 10, vol. 02, p. 05-15. octobre 2019. ISSN: 2448-0959,

Lien

d'accès: https://www.nucleodoconhecimento.com.br/sante/massotherapie-avec-

$\underline{\text { balles }}$

\section{RÉSUMÉ}

La massothérapie a été utilisée au logo de l'existence de l'humanité à des fins diverses et il existe différents types de massage allant du classique au transcendantal. Parmi les différentes modalités de massage il ya massage avec des boules. II s'agit d'un massage très efficace et peu abordé dans la littérature actuelle, de sorte que la

${ }^{1}$ Master en Sciences de la Motricité Humaine; Spécialisation en physiothérapie dermato-fonctionnelle; Spécialisation en entraînement sportif et en entraînement personnel; Spécialisation en Gymnastique Médicale; Spécialisation dans les sujets avancés en physiothérapie; Spécialisation en portugais; Spécialisation dans l'enseignement innovant de l'enseignement supérieur; Diplômé en physiothérapie; Technologue en esthétique et cosmétiques; Baccalauréat et Baccalauréat en Éducation Physique; Diplômé en Lettres; Diplômé en pédagogie.

2 Master en Sciences de la Motricité Humaine; Spécialisation en Gymnastique Médicale; Diplômé en physiothérapie; Baccalauréat et Baccalauréat en Éducation Physique. 
présente étude a été menée et visait à étudier l'origine de ce massage et de décrire les techniques d'application. À cette fin, une recherche bibliographique basée sur des livres et des bases de données obtenus chez Scielo et Scholar Google a été menée. Il a été constaté que ce type de massage a été effectué dans la dynastie Ming et en plus du massage pourrait être effectué des exercices avec les boules. II ya essentiellement quatre techniques de massage comme les balles.

Mots-clés: massage, massage, massage comme boules.

\section{INTRODUCTION}

L'une des thérapies les plus anciennes qui a été utilisé à ce jour est la massothérapie et il a des fins différentes telles que la diminution des tensions, la libération de toxines, promouvoir la relaxation musculaire, la fatigue de combat et de stimuler la circulation sanguine. (CANNECCHIA, 2019).

Le massage est une pratique courante tout au long de l'histoire de l'humanité et a été largement abordé dans la littérature. Aujourd'hui, il existe plusieurs vidéos de massothérapie qui peuvent être facilement accessibles sur Internet, sur Facebook, Instagram, YouTube, WhatsApp, etc. Cependant, il est nécessaire de souligner que tout ce qui est affiché dans les médias numériques n'est pas correct et cela crée beaucoup de confusion pour les laïcs et les débutants dans le monde de l'esthétique. Par conséquent, il est important de développer des études qui permettent la divulgation appropriée des différentes techniques de massothérapie et de ses avantages réels.

On sait qu'il existe différents types de massothérapie, tels que: suédois, thaïlandais, hawaïen, tantrique, indien, etc, mais l'une des techniques les moins décrites est le massage avec des balles, ou tout simplement massage avec des balles. Il s'agit d'une technique de massage qui est appliquée sur les tissus mous et exerce trois effets de base: mécanique, physiologique et psychologique. Son application suit des principes similaires à la massothérapie suédoise, cependant, en utilisant des balles pour masser la région du corps. 
Il est connu qu'il ya un manque de littérature scientifique sur l'utilisation de massage de balle, de sorte que la présente étude a été menée et visait à étudier l'origine de ce massage et de décrire les techniques d'application. À cette fin, une recherche bibliographique basée sur des livres et des bases de données obtenus chez Scielo et Scholar Google a été réalisée et a eu comme descripteurs: massage, avantages du massage, massage comme boules.

\section{EXAMEN DE LA LITTÉRATURE}

\subsection{HISTOIRE DE MASSAGE}

Il existe des documents historiques qui prouvent que même les premières civilisations sauvages, en plus des civilisations civilisées, pratiquaient déjà un certain type de massage, en particulier la friction (WOOD et BECKER, 2008). C'était une pratique courante dans les anciennes civilisations indienne, chinoise, japonaise, grecque et romaine. On sait que l'une des plus anciennes références apparaît dans Nei Ching qui est un texte médical chinois qui a été écrit dans une période avant 1500 avant JC. Plus tard, des écrits ont été trouvés sur le massage par les médecins hippocrate au 5ème siècle avant JC. et Avicenne et Ambrose Stop, respectivement, aux Xe et XVle siècles. (CASSAR, 2001).

Cependant, les Égyptiens ont déjà appliqué le massage à des fins thérapeutiques à plus de 4000 a. C. Et en Inde, le massage a cherché une vision holistique pour équilibrer le corps, l'esprit et l'esprit et d'être l'une des recommandations et des enseignements. On sait que la description du massage se trouve dans les livres Ayur Veda qui ont été écrits à environ 1800 avant JC. Cependant, il est connu que presque toutes les grandes cultures du monde ont proposé en détail les indications et les avantages du massage qui a été généralement combiné avec d'autres types de traitement traditionnel, en particulier les traitements de bain. (SILVA, 2016).

Il est nécessaire de souligner qu'Hippocrate (480 av. J.-C.) a appelé le massage de l'anatripsis, dont le sens est «rubbing en appuyant sur le tissu», plus tard, le terme a été traduit en latin mot frictio, qui signifie «friction» ou «frottement». On sait que ce 
terme a prédominé pendant longtemps et a été utilisé aux États-Unis jusqu'en 1870. En Inde, le massage a été appelé shampoing; en Chine, Cong-Fou; au Japon, Ambouk (CASSAR, 2001).

À son tour, Hippocrate (460-370 av. J.-C.) a également utilisé le massage comme traitement, parce qu'il croyait que le corps est capable de s'auto-guérir s'il est correctement stimulé. Asclepâdes de Bitila était un autre médecin grec qui utilisait des massages, des régimes et des exercices pour traiter les maladies. En outre, il est notoire de savoir que les civilisations primitives australienne, égyptienne, russe, ukrainienne, du Pacifique et du Nord et du Sud ont utilisé le massage par des mouvements de friction avec des huiles et des eaux comme un moyen de chasser les démons et les esprits qui ont généré des maladies chez les gens et ainsi purifié le corps des malades. Par conséquent, plusieurs personnes ont utilisé et recommandé le massage au profit de la santé des humains. (BERTOJA e TOKARS, 2018).

Cependant, le massage n'a pas toujours été bien considéré, avec la montée du christianisme au Moyen Age (période historique allant du 5ème siècle au 15ème siècle), le culte du corps est venu à être considéré comme un péché. Par conséquent, le soin d'hygiène n'est plus pris et donc le massage a été interdit dans les civilisations à crête. (BRAUN et SIMONSON, 2007). En Europe, l'Église catholique considérait le massage comme un péché et son association avec les herbes médicinales était considérée comme un acte de sorcellerie. (RAMOS, 2017).

Avec la fin du Moyen Age et l'émergence de la Renaissance, le massage a été découvert et appliqué à nouveau et plusieurs auteurs ont contribué à ce fait. Cependant, c'est entre 1776 et 1839 que Pehr Henrik Ling révolutionne la pratique des exercices et du massage. II a créé les terminologies et la technique du massage suédois qui est également connu sous le nom de massage classique ou de guérison par le mouvement suédois. Sa technique a d'abord été popularisée en Europe et en Russie, puis mondialisée (WOOD et BECKER, 2008).

C'est également Hippocrate qui a décrit les avantages du massage associé aux propriétés chimiques des huiles utilisées (FRITZ, 2002). Par conséquent, il peut être 
vu que non seulement le massage pourrait apporter des avantages, mais aussi la propriété chimique de certaines huiles végétales et essentielles.

Le mot massage provient de la masse grecque qui signifie " pétrir » qui signifie toucher, manipuler, serrer (FRITZ, 2002). Cependant, le terme massothérapie est actuellement préférable, puisque le mot thérapie indique l'utilisation thérapeutique du massage. Aujourd'hui, il existe plusieurs techniques de massage parmi elles : détente, sport, thérapeutique, modélisation, massage rapide, réflexologique, tantrique, etc. Tout comme plusieurs types d'équipements ont émergé qui aident le thérapeute à appliquer le massage, parmi eux sont les boules de baoding ou boules de santé.

Les boules de baoding (figure 1) sont d'origine chinoise et leur dénomination vient de la ville de baoding, située dans la province du Hebei en République populaire de Chine. Ce sont des artefacts utilisés pour exercer les mains et fournir de la relaxation, diminuant le stress. Traditionnellement, ils sont composés d'une paire de boules de fer de petite taille, qui sont stockées dans une petite boîte rectangulaire. Ces boules proviennent de la dynastie Ming (1368-1644 après JC) et ont été faites par un artisan d'armes guidé par un rêve céleste. II a créé deux boules de fer basées sur le concept yin/yang : l'une représentant le rugissement du Dragon et l'autre le coin du Phénix.

Au début, l'empereur de Chine a été charmé par les avantages thérapeutiques fournis par les boules et en a fait un instrument qui ne pouvait être utilisé que par la famille royale. Plus tard, les boules de fer sont devenues populaires et ont atteint le monde. Initialement, ils ont été utilisés pour améliorer la condition physique et le tempérament de train (diminuer l'anxiété, calmer) but pour lequel ils sont encore utilisés aujourd'hui. Ils sont un appareil pour détendre les muscles et les articulations et pour la défense dans la pratique des arts martiaux.

De nos jours, les boules de baoding sont faites de plusieurs types de matériaux, mais restent légères à transporter. Ils sont le plus souvent creux et faits d'acier chromé et contiennent des guizos à l'intérieur. Les guizos fournissent des sons qui peuvent sonner comme des cloches relaxantes qui répond à la nature et l'influence des cinq doigts, donc l'exercice des muscles et activer la circulation sanguine. 
Figure 1: Balls baoding.

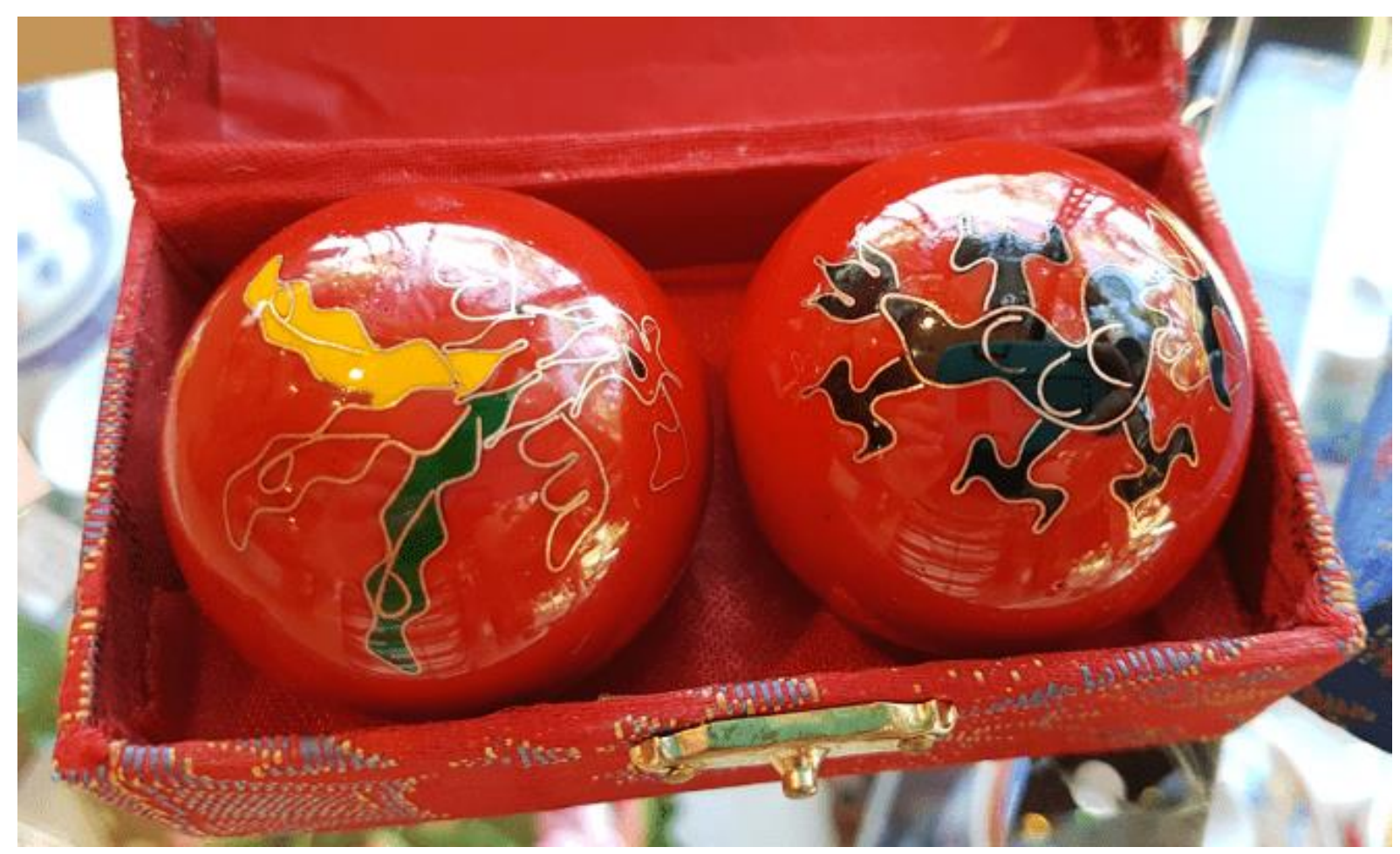

Fonte: Os autores

Avec le développement de nouveaux matériaux et l'évolution de la technique, les boules ont commencé à être produites avec des matériaux magnétiques, du bois, du silicone et du métal, du caoutchouc, du silicone, etc. (Figure 2)

Figure 2 : Différents types de boules.
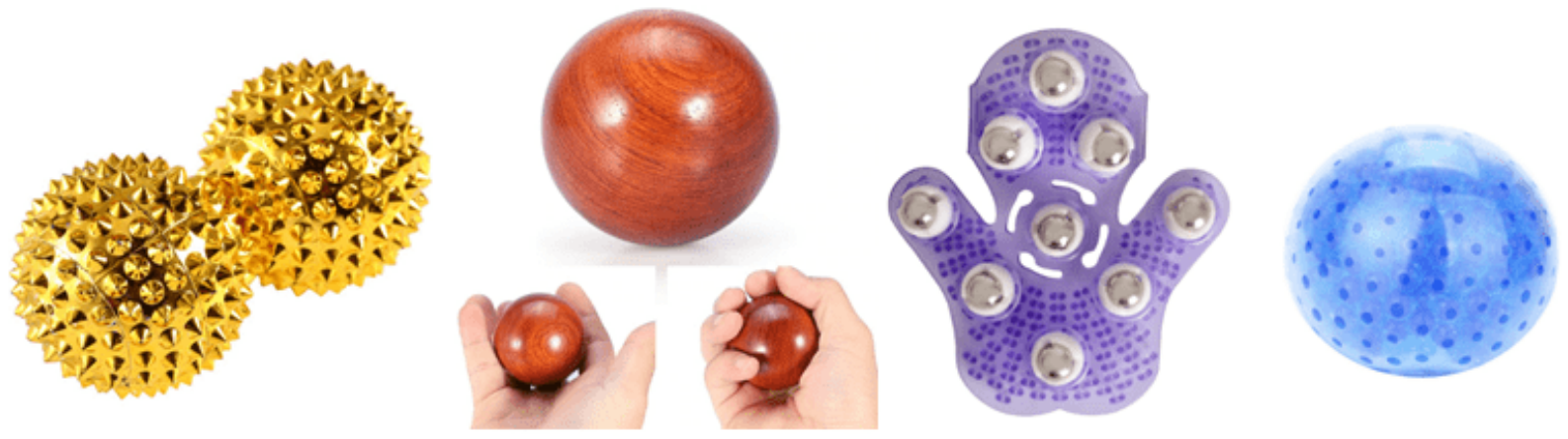

Fonte: Os autores 
Quand les boules de fer sont apparues, elles étaient uniques au massage de leurs mains. La personne s'auto-massaged en tournant les boules entre ses doigts. Cette technique est encore largement utilisée, mais il est possible de masser toutes les parties du corps avec l'aide du thérapeute ou même diverses parties du corps par l'auto-massage. (figure 3)

Figure 3 : Automassage avec balle.
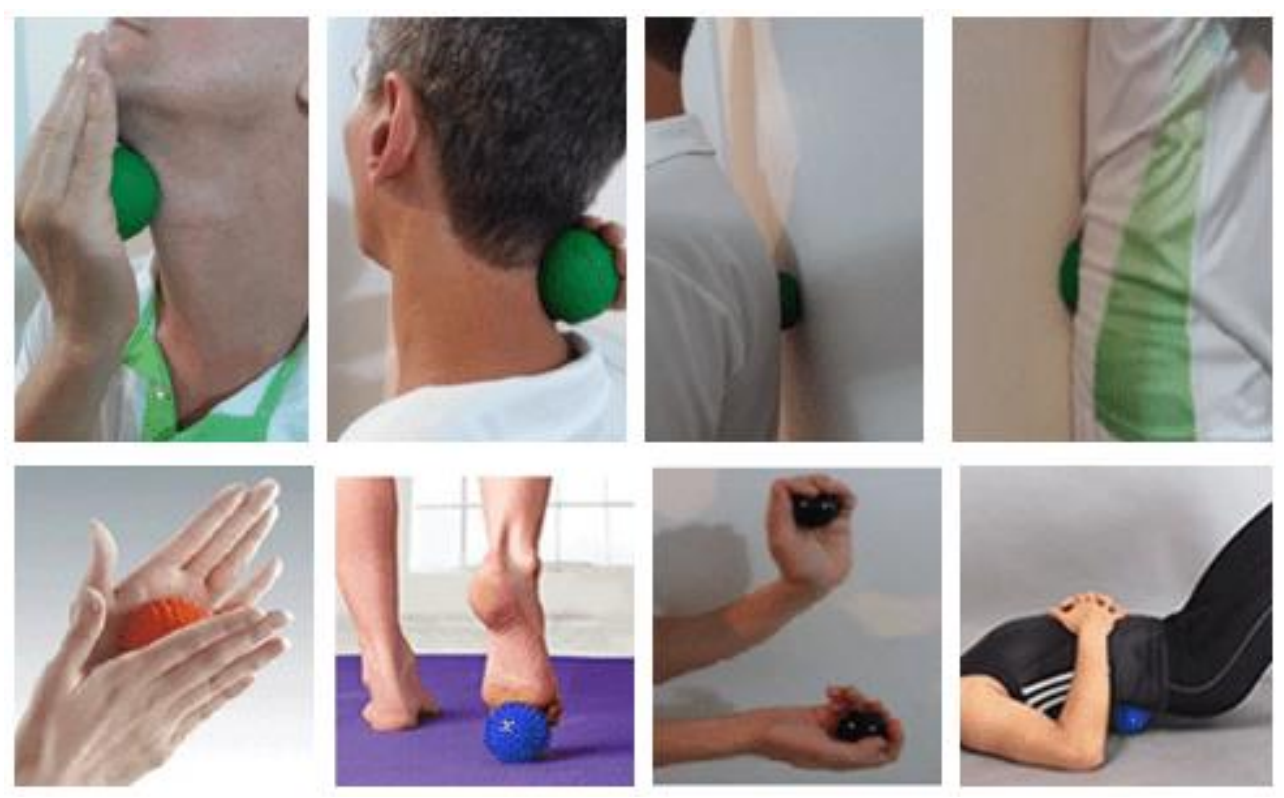

Fonte: Os autores

\subsection{MASSAGE DE BOULE : AVANTAGES}

Le massage à la balle (massage à la balle) est appliqué sur les tissus mous et exerce trois effets de base : mécanique, physiologique et psychologique. Les principaux effets du massage sont mécaniques, mais ils génèrent des effets physiologiques et psychologiques.

Le massage est une technique qui favorise la manipulation sur les tissus mous et comme tout type de massage relaxant, il permet la diminution des tensions musculaires, en plus de stimuler la production de sérotonine et d'histamine qui ont l'action vasodilatateur, augmentant sang et l'apport lymphatique. Le massage peut 
activer les rmorécepteurs et augmenter la perméabilité de la membrane cellulaire, ce qui permet l'absorption des nutriments et, par conséquent, une meilleure nutrition cellulaire, l'oxygénation et l'hydratation (CLAY, 2008).

Psychologiquement, le massage de boule est capable de fournir la relaxation de muscle et mentale, causant un état enclin au sommeil. On sait que le massage stimule la libération d'hormones endorphines et de sérotonine qui sont responsables de la sensation de plaisir. (PEREIRA, 2013). Les hormones d'endorphine et la sérotonine permettent la relaxation et en même temps sont ceux responsables du sommeil profond, qui est désigné avec le sommeil paradoxal. En outre, le massage réduit le cortisol qui est l'un des responsables de l'insomnie / stress, de sorte qu'une personne qui reçoit le massage aura moins de cortisol dans la circulation sanguine, ce qui entraînera une amélioration de leur qualité de sommeil et la diminution de l'anxiété et le stress.

L'étirement, la compression, la traction et les mouvements de frottement qui sont effectués dans le massage de boule exercent des forces mécaniques évidentes sur les tissus. Ces forces mécaniques exercent des effets mobilisateurs d'adoucissement et d'étirement sur la peau, les tissus sous-cutanés, les viscères et les muscles.

L'effet mécanique se rapporte aux influences directes que le massage joue sur les tissus mous étant massés (CASSAR, 2001). II fournit des effets physiologiques sur les systèmes musculaire, nerveux, circulatoire et digestif. Massage avec des boules lors de l'élimination lente des muscles stimule le réflexe tendineux qui réduit la tension musculaire et entraîne une relaxation musculaire. Avec la relaxation de muscle squelettique et les manoeuvres coulissantes faites avec des boules il est possible d'exercer assez de pression pour déplacer les liquides dans la direction du flux circulatoire veineux ou lymphatique. Si les manœuvres sont vigoureuses, elles peuvent entraîner des augmentations significatives du flux sanguin dues à des phénomènes de vasodilatation et de capillatisation. Ces effets circulatoires stimulent les échanges entre les médias cellulaires et sanguins, améliorent l'oxygène et l'apport nutritionnel, aident à éliminer les résidus du métabolisme et le dioxyde de carbone. Les manœuvres avec une pression plus suable, rythmique et appliquée vers les 
chemins des vaisseaux lymphatiques superficiels permettent d'accélérer le flux de retour lymphatique, étant utile en cas d'œdème pour aider à la résorption.

Les principaux effets mécaniques produits par le massage sont : la mobilisation du sang veineux, de la lymphe, de l'œdème et de l'hématome, du contenu intestinal, du mucus, des fibres musculaires et de la masse, des tendons, de la peau et des tissus sous-cutanés, des adhérences et des tissus cicatriciels (Gouvernement de l'État de la Cear, 2010).

Nous soulignons également les effets physiologiques du massage de boule sur le système digestif qui améliorent le transit intestinal. Le massage stimule le péristaltisme pour favoriser l'évacuation des appartements et des excréments du gros intestin, facilitant la fonction excrétrice en cas de constipation intestinale.

Sans aucun doute la réduction de la douleur lors de l'application du massage est obtenue par différents mécanismes, parmi la diminution des spasmes musculaires et l'œdème. Par conséquent, l'amélioration de la circulation de retour absorbe l'oœdème et avec lui les métaboliques cellulaires. II réduit également en activant les récepteurs cutanés qui inhibent la douleur par le mécanisme de la porte (STARKEY, 2017).

La relaxation musculaire et l'amélioration de la circulation contribuent déjà à diminuer les algiques musculo-squelettiques. Cependant, le massage de boule réduit la douleur parce qu'il obéit aux phénomènes de la théorie d'inondation. Cette théorie nous permet de comprendre qu'il existe différents types de fibres nerveuses et chacun a une vitesse de conduite de l'impulsion nerveuse et une fonction. Dans ce cas, la fibre nerveuse de douleur est plus lente que la fibre de nerf de tact et de pression. Par conséquent, quand une personne ressent une douleur musculaire et est massée, les impulsions nerveuses du toucher et de la pression arrivent d'abord dans la moelle épinière et inhibent les voies nerveuses de la douleur qui conduirait l'information de douleur au thalamus (site du cerveau qui permet de sentir que de la r).

L'effet du massage sur le contrôle de la douleur est si important qu'il est utilisé comme soins palliatifs chez les patients atteints d'un cancer à un stade avancé. Un fait qui se 
trouve dans la déclaration de florentine (2012, n.p.) qui rapporte que "le massage est une technique utilisée comme thérapie complémentaire chez les patients atteints de cancer, dans le but de fournir un soulagement de la douleur."

Il convient de souligner que pour que les effets inhibiteurs se produisent, il est nécessaire que les manœuvres de massage avec des balles sont faites constamment, lentement et en douceur, de sorte que l'accommodement se produit en augmentant le seuil de perception du nouveau stimulus.

Le massage de boule aide dans le renouvellement des cellules épidermiques et permet aux glandes sébacées d'être claire et de fonctionner mieux. Et en raison des phénomènes vasodilatateurs et de la capillisation, la peau devient plus nourrie et oxygénée, en plus de faciliter la perméation de certains actifs cosmétiques.

La massothérapie peut exercer une influence sur le système immunitaire, car elle agit sur les couches de la peau qui réagit positivement. (CARVALHO e ALMEIDA, 2018).

\subsection{MASSAGE BALL: ORIENTATIONS, INDICATION ET CONTRAINDICATION}

Avant d'appliquer le massage avec des balles, il est nécessaire pour le thérapeute de faire une évaluation (anamnésie) du client. L'évaluation fournira des informations utiles pour choisir si oui ou non la personne à masser, et permet de sélectionner les manœuvres prioritaires à appliquer. Au cours de l'évaluation, si le client présente une contre-indication relative, il devrait être chargé de consulter un autre professionnel de la santé et ne peut être massé que s'il a une autorisation écrite du médecin ou du physiothérapeute.

Les principales données qui devraient être présentes dans le formulaire d'évaluation sont les données d'identification (nom complet, adresse, numéro de téléphone, date de naissance, état matrimonial et profession), les données sur l'état de santé (si la personne est en bonne santé ou a une maladie : diabète, hypertension ou hypotension, maladie cardiaque, cancer, migraine, épilepsie, constipation, dysménorrhée, cystite, 
allergies, insomnie, dépression, etc.) et il est nécessaire de savoir si la personne est enceinte ou allaite. D'autres renseignements qui peuvent contenir dans le formulaire d'évaluation sont sur la pratique de l'exercice physique, l'alimentation, la consommation de boissons alcoolisées, le tabac et les médicaments en usage.

L'application de la massothérapie de boule prête l'attention aux mêmes contreindications de n'importe quelle application de massage et elles peuvent être absolues, c'est-à-dire, le massage ne peut pas être appliqué au client ; ou relative, c'est-à-dire, il est possible d'appliquer un massage, mais certains soins doivent être adoptés ou certaines zones ne peuvent pas être massées.

Les principales contre-indications absolues sont la thrombose ou des lésions vasculaires instables, l'état fébrile, la présence d'infection, la gangrène, les maladies rénales, les maladies cardiaques avancées, les maux de tête graves, les changements incontrôlés de la pression artérielle par les médicaments, Intoxication. Les contreindications relatives sont : fractures (avant solidifiées), brûlures récentes, plaies ouvertes. (VERSAGI, 2015). D'autres contre-indications relatives sont : le cancer, la maladie mentale, les décalcifications et la grossesse.

Les principales indications sont: soulagement du stress, constipation, migraine, récupérer la personne en cas de blessures traumatiques (entorse, ecchymoses) après la libération médicale, réduire les tensions musculaires et tendineuses, myalgies par l'effort, la prévention des MST, drainage de l'œdème veineux et lymphatique, adjuvant dans le traitement des vergetures, lipodystrophie localisée, panique d'eedème fibrosclérotique, insomnie, anxiété, etc.

\subsection{MASSAGE DE BOULE : TECHNIQUES}

Certaines règles de base pour l'application du massage doivent être suivies, telles que: le massothérapeute et le client doivent se sentir à l'aise pendant la session; le contact avec le client ne doit pas être soudainement supprimé; le massage doit être interrompu dans les régions où le patient ressent de la douleur; ne jamais appuyer ou masser directement sur la colonne vertébrale. (ELLSWORTH, 2012). 
Les manœuvres de massage à billes doivent respecter les principes de direction, de pression, de vitesse, de rythme et de durée selon les objectifs souhaités.

La direction dépendra de l'effet désiré, de sorte qu'il peut être effectué dans le sens de la circulation qui est souhaité pour stimuler ou dans la direction des fibres musculaires à masser. En ce qui concerne le débit artériel, les manœuvres doivent être des centrifugeuses ou quasi-distal pour masser les membres, le tronc et le cou et centripète ou proximal pour masser la tête. Si la direction du débit est veineuse, les manœuvres doivent être centripètes ou proximales pour masser les membres, le tronc et le cou et les centrifugeuses ou quasi-distal pour masser la tête. Si vous visez le drainage lymphatique, il est nécessaire de suivre la direction de l'écoulement des vaisseaux lymphatiques et de maintenir la séquence de drainage proposée par José Maria Pereira de Godoy et Maria de Fàtima Guerreiro Godoy.

Quant à la pression à exercer, il doit être confortable et agréable lors de la recherche de relaxation musculaire et la réduction de la douleur. II est à noter qu'il est nécessaire d'obtenir des commentaires des clients pour que la pression soit ajustée. II y a des personnes plus musclées ou obèses où la pression aura tendance à être légèrement plus élevée par rapport à maigre, les personnes âgées ou les enfants. Si vous visez le drainage lymphatique, la pression doit être comprise entre 30 et $40 \mathrm{mmHg}$.

La vitesse et le rythme varient également en fonction des besoins. Les manœuvres de relaxation et de drainage doivent être sans rythme lent, constant et bien rythmé. Pour la dynamisation circulatoire artérielle, le traitement de striation, la lipodystrophie localisée, le fibroedemageloid devraient être plus rapides. II est bon de se rappeler que les mouvements effectués lentement ont tendance à être relaxant, tandis que l'inverse, plus stimulant.

Le massage dure de 5 à 20 minutes lorsqu'il est appliqué dans une seule région, sauf si l'objectif est le drainage lymphatique, dans ce cas, il durera de 30 à 40 min. Lorsqu'il est appliqué d'une manière systémique, c'est-à-dire dans l'ensemble du corps, visant à la relaxation musculaire et à la diminution des tensions émotionnelles, il durera de 45 à 90 min. 
Il convient de noter que le temps dépendra fortement de l'objectif à atteindre, et sur la taille de la zone du corps, car il faut moins de temps pour traiter une personne relativement petite par rapport à une grande personne. Chez les personnes très jeunes et très âgées, la durée du massage doit être réduite, parce que l'arc réflexe est plus sensible et l'effet intégral est atteint plus rapidement.

Le massage à billes appliqué par le thérapeute est divisé en quatre manœuvres de base et chaque manœuvre a ses variations. La manœuvre qui initie et termine le service est celle du glissement, qui consiste à glisser vers ou avec les boules sur la surface du corps du client toujours en réponse à l'écoulement circulatoire souhaité ou la direction des fibres musculaires ou le sens du péristaltisme (Figures 4 à 11).

Figure 4 : Technique de glissement longitudinal symétrique dans Mmli.

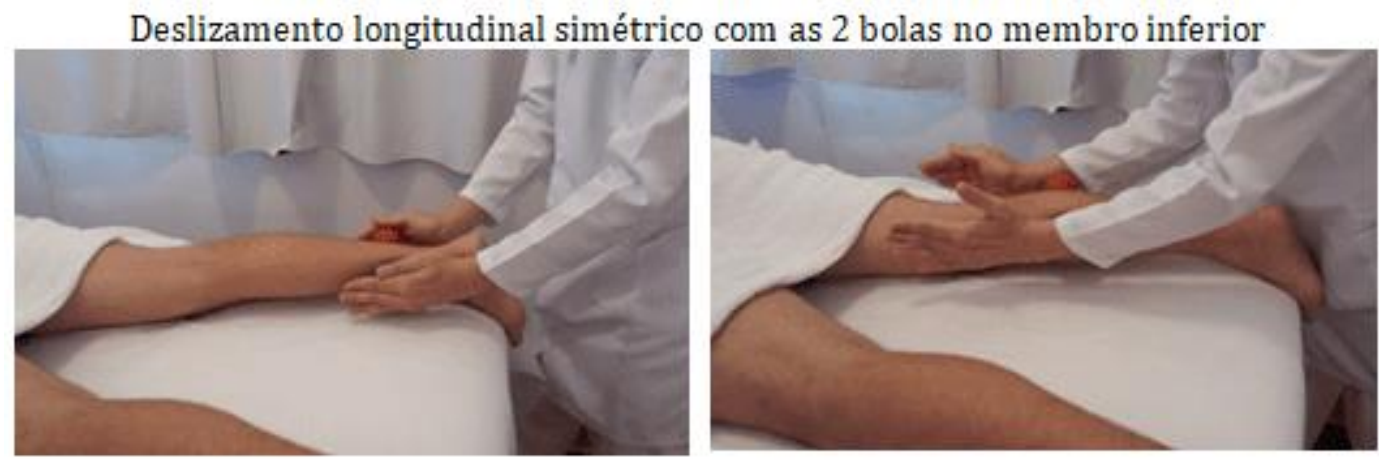

Fonte: Os autores

Figure 5 : Technique de dérapage longitudinal asymétrique dans le coffre.

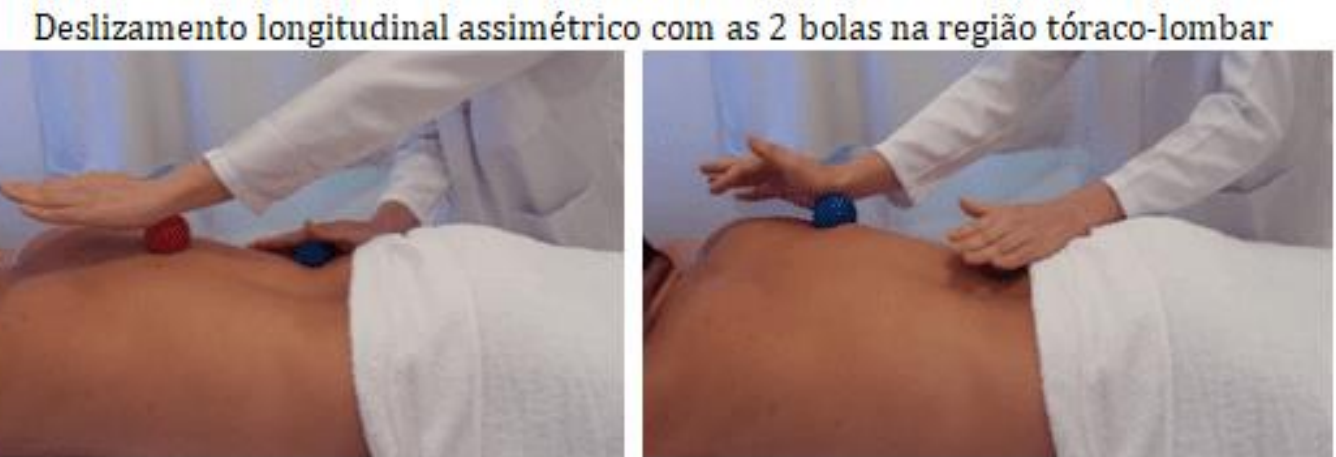

Fonte: Os autores 
Figure 6 : Technique de glissement croisé.

\section{Deslizamento transversal com as 2 bolas na região tóraco-lombar}
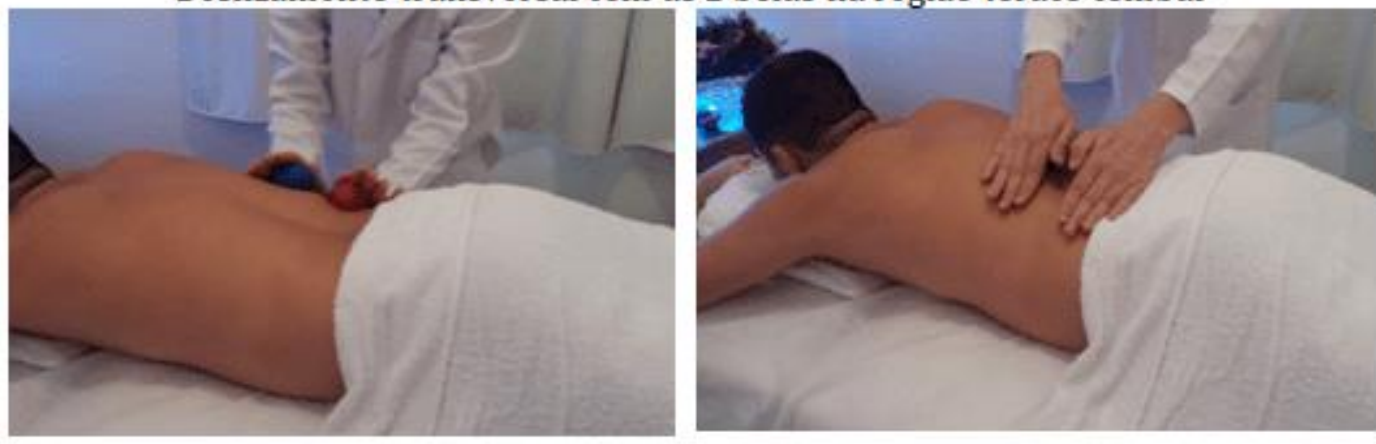

Fonte: Os autores

Figure 7 : Technique de dérapage longitudinal symétrique dans le tronc.

\section{Deslizamento longitudinal simétrico com as 2 bolas na região tóraco-lombar}
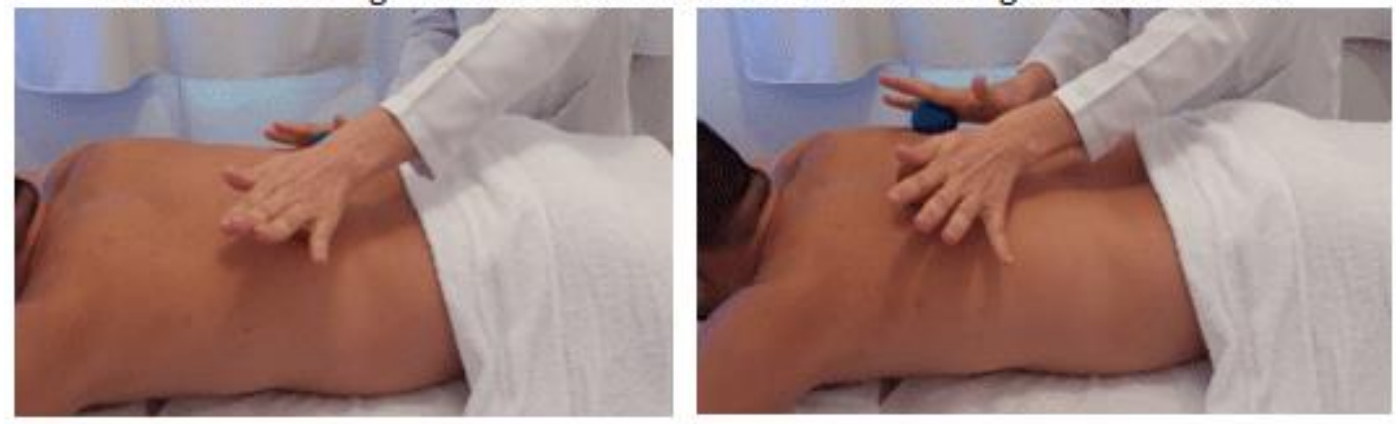

Fonte: Os autores

Figure 8 : Technique de glissement circulaire.

Deslizamento circular com uma bola na região abdominal no sentido do peristaltismo.

A manobra pode ser executada com uma ou duas mãos.

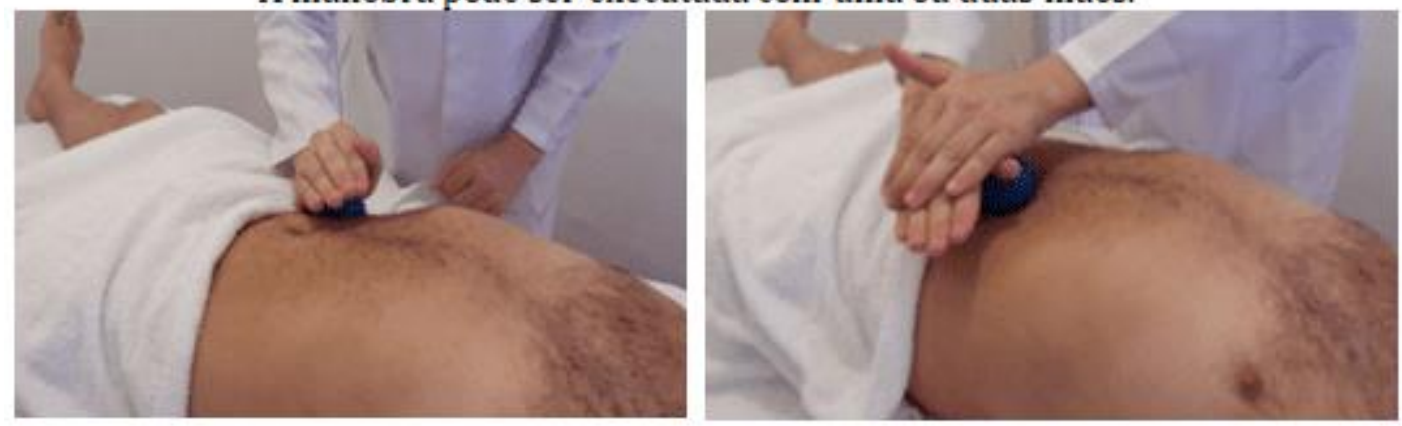

Fonte: Os autores

$\mathrm{RC}: 41887$

Disponível em: https://www.nucleodoconhecimento.com.br/sante/massotherapie-avec-balles 
Figure 9 : Technique de dérapage longitudinal symétrique sur l'avant-bras.

Deslizamento longitudinal simétrico com as 2 bolas na mão e no antebraço
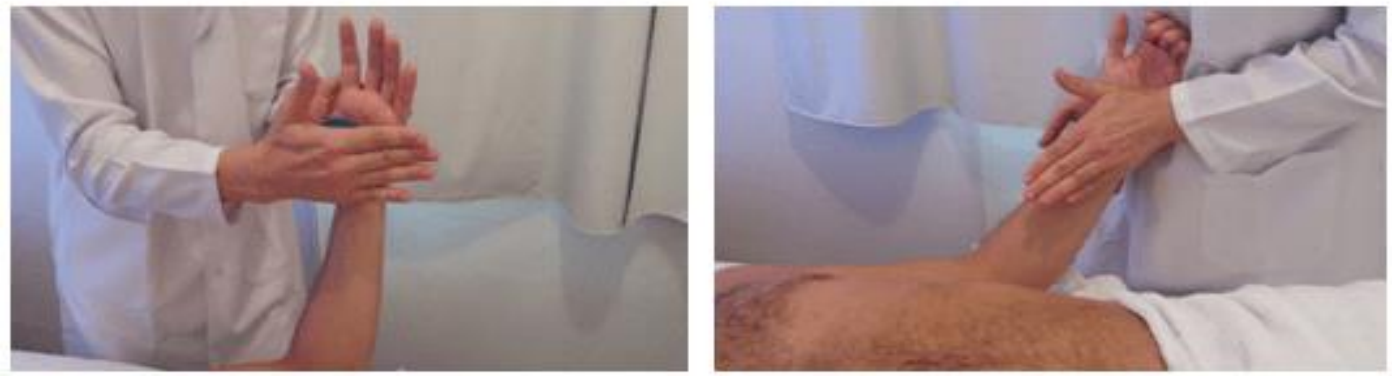

Fonte: Os autores

Figure 10 : Technique de glissement longitudinal symétrique sur le bras.

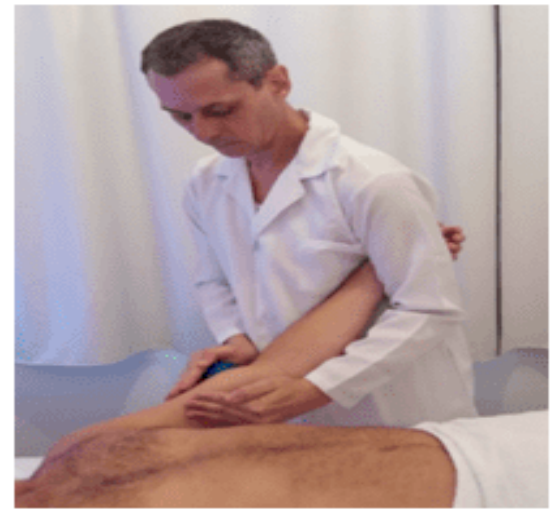

Deslizamento longitudinal simétrico com as 2 bolas no braço. Observe que o terapeuta sustenta $\circ$ membro superior fixando-o entre o tronco e o braço. É fundamental que o cliente não sustente o membro.

Fonte: Os autores

Figure 11 : Technique de glissement diagonale.

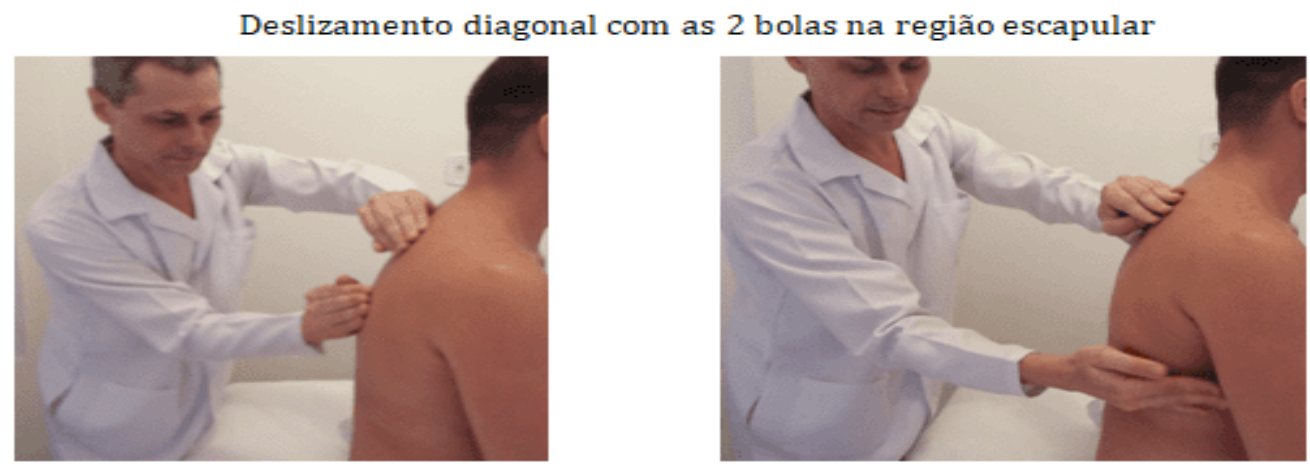

Fonte: Os autores

RC: 41887

Disponível em: https://www.nucleodoconhecimento.com.br/sante/massotherapie-avec-balles 
La deuxième manœuvre est la compression fixe ou la compression et la traînée. La première consiste à maintenir la région comprimée par la balle pendant 10 à 15 secondes ou tout au long de la session. La seconde consiste à compresser la balle contre le corps et à la traîner en mouvements circulaires (figures 12 et 13).

Figure 12 : Technique de compression dans le pied.

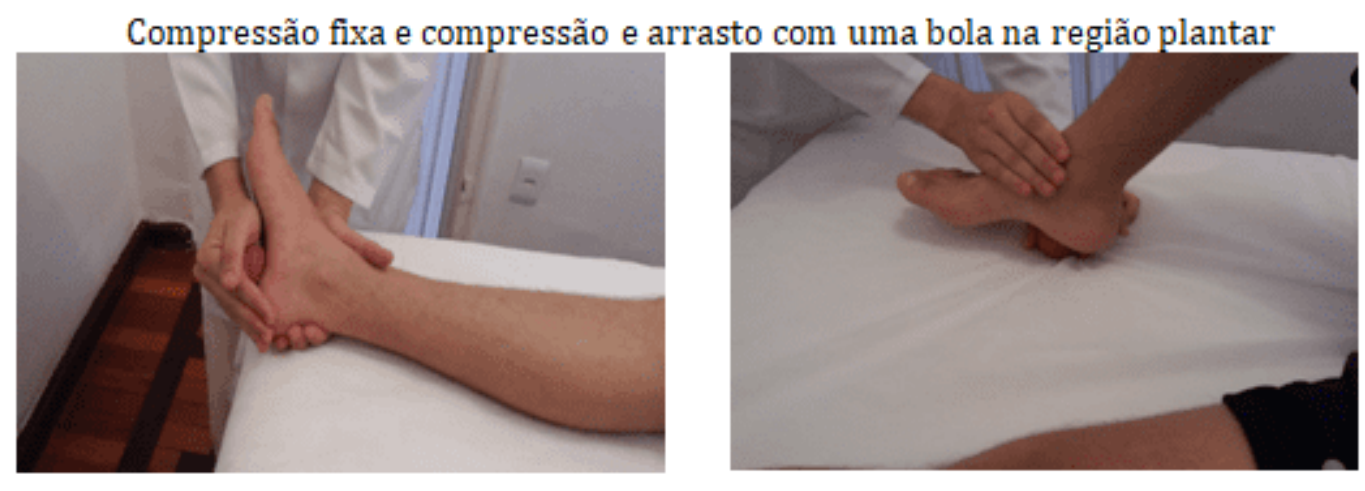

Fonte: Os autores

Figure 13 : Technique de compression dans la colonne vertébrale.

Compressão fixa com as 2 bolas na região paravertebral cervical e lombar
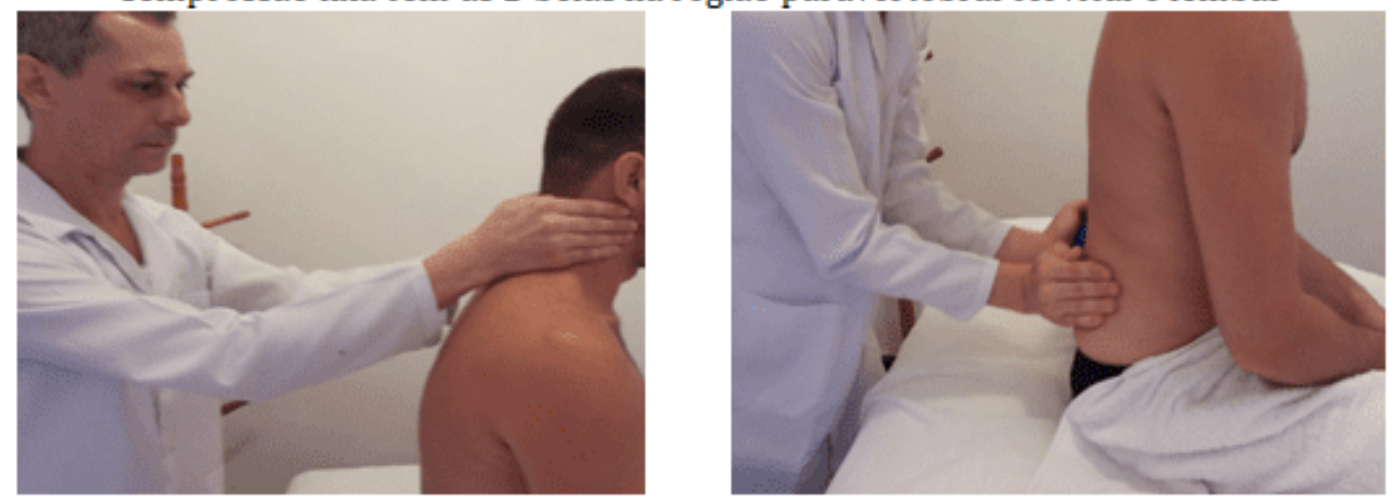

Fonte: Os autores

La troisième manœuvre est le pétrissement et dans ce cas deux boules sont toujours utilisées. Le thérapeute pétrit le tissu mou entre les boules avec une belle pression ou une forte pression, mais pas mal à l'aise. Le client ne peut signaler de douleur ou de peine dans une hypothèse (figure 14). 
Figure 14 : Technique de pétrissement.

\section{Amassamento de trapézio e da loja posterior de perna}
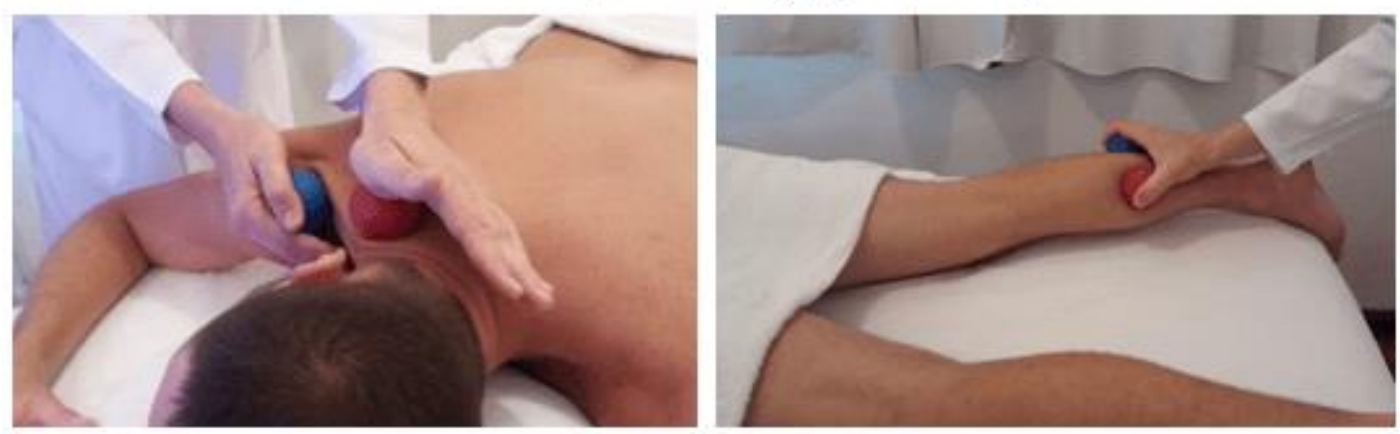

Fonte: Os autores

La quatrième manœuvre de base est le frottement et dans ce cas, il est généralement utilisé dans les petites régions et avec une seule balle. Cette manœuvre est une sorte de glissement profond, effectué vigoureusement et rapidement, afin de réduire les nodules de tension musculaire, défaire les caillots, augmenter la microcirculation locale. Provoque un grand chauffage de la région massée et est recommandé en cas de stries et de lipodystrophie localisée. Les manœuvres visent à chauffer la région et se font avec la balle circulant entre la paume et la surface du corps du client (figure 15).

Figure 15 : Technique de friction.

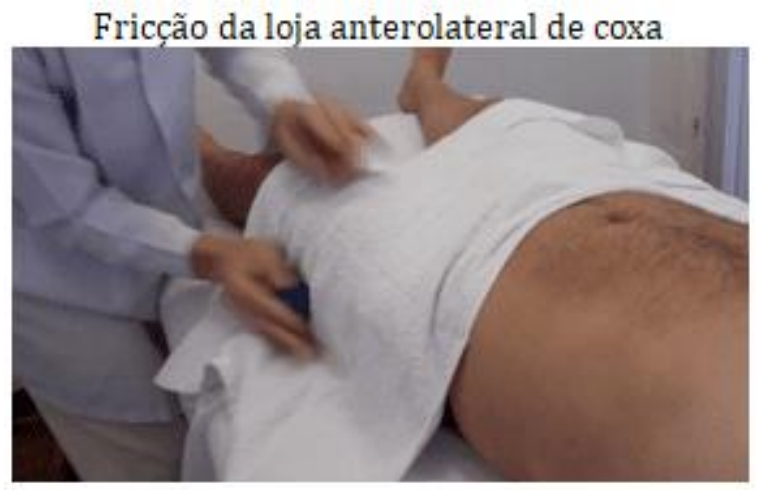

Fonte: Os autores 
Il ya des variations de chacune des manœuvres de base, en plus de la technique de massage auto avec des balles, que la personne applique le massage sur elle (figure 16).

Figure $16:$ Technique d'automassage.

\section{Self Ball Massage}

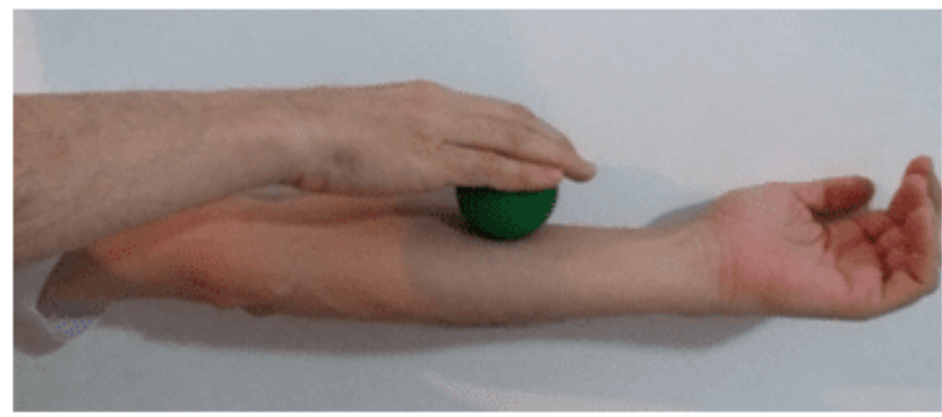

Fonte: Os autores

\section{CONCLUSION}

Les premiers enregistrements historiques de la massothérapie remontent à 1800 av. J.-C. et se trouve dans les livres Ayur Veda, mais il est connu que les Egyptiens ont utilisé le massage à des fins thérapeutiques pour plus de 4000 a. C. E c'est dans la dynastie Ming (1368-1644 ap. J.-C.) qu'un artisan d'armes a créé deux boules de fer basées sur le concept yin/yang. Ils ont commencé à être employés pour fournir des effets thérapeutiques et pour améliorer la condition physique et le tempérament de train (diminuer l'anxiété, se calmer).

Aujourd'hui, les boules de fer sont encore utilisées, mais d'autres matériaux ont émergé avec du bois, du silicone, du caoutchouc et plusieurs autres. Toutes ces boules, quel que soit le matériel, servent à masser et à faire de l'exercice et peuvent fournir des bienfaits organiques et psychologiques déjà reconnus en massothérapie. On peut voir que le massage de balle appliqué par le thérapeute est divisé en 4 manœuvres de base, mais chaque manœuvre a ses variations. 


\section{RÉFÉRENCES}

BERTOJA, Vanessa Gomes Bertoja; TOKARS, Eunice. Os benefícios da massagem relaxante.

em:

https://www.librarybus.com/view?t=0s+benef\%C3\%ADcios+da+massagem+relaxant e+-+TCC+On-line+-

+Tuiuti\&u=http\%3A\%2F\%2Ftcconline.utp.br\%2Fmedia\%2Ftcc\%2F2017\%2F05\%2F OS-BENEFICIOS-DA-MASSAGEM-RELAXANTE.pdf. Acesso em: 10 jan.2018.

BRAUN, Mary Beth; SIMONSON, Stephanie. Massoterapia. São Paulo: Manole, 2007.

CANNECCHIA, Marcela Cleto. Benefícios da massagem relaxante para o homem da atualidade. Pesquisa e Ação. Mogi das Cruzes, SP. v.5, n.1, p. 46-49. Jun. 2019. Disponível em: https://revistas.brazcubas.br/index.php/pesquisa/article/view/574/703. Acesso em: 05 ago. 2019.

CARVALHO, Rosemeire de Jesus; ALMEIDA, Maria Antonieta Pereira Tigre. Efeitos da Massoterapia sobre o Sistema Imunológico. Id on Line Rev. Mult. Psic. v.12, n. 40. p.353-366, 2018

CASSAR, Mario-Paul. Manual de Massagem Terapêutica: um guia completo de massoterapia para o estudante e para o terapeuta. São Paulo: Manole, 2001.

CLAY, James. H.; POUNDS, David. M. Massoterapia Clínica: integrando anatomia e tratamento. São Paulo: Manole, 2008.

ELLSWORTH, Abigail. Massagem: anatomia ilustrada, guia completo de técnicas básicas de massagem. Barueri, São Paulo: Manole, 2012.

Florentino, Danielle de M. A fisioterapia no alívio da dor: uma visão reabilitadora em cuidados paliativos. Brazilian Journal of Health and Biomedical Sciences. Rio de Janeiro; Revista do Hospital Universitário Pedro Ernesto. v.11, n.2, abr/jun, 2012. 
FRITZ, Sandy. Fundamentos da Massagem Terapêutica. 2.ed. São Paulo: Manole, 2002.

GOVERNO DO ESTADO DO CEARÁ. Técnico em Massoterapia: técnicas clássicas e modernas de massoterapia. Escola Estadual de Educação Profissional - Ensino Médio Integrado à Educação Profissional - Curso Técnico em Massoterapia. Fortaleza Ceará, 2010.

PEREIRA, Maria de Fátima Lima. Recursos Técnicos em Estética. Vol. I. Série Curso de Estética. São Paulo. Difusão Editora. 2013.

RAMOS, Andréa Rosa. Técnicas de massagem corporal. Londrina: Editora e Distribuidora Educacional S.A, 2017

SILVA, Carina Geremias dos Santos. Massoterapia Mãos de Anjo. Plano de Negócios. PG, Gravataí, v. 2, n. 2, 2016. Disponível em: http://revista.faqi.edu.br/index.php/PN/article/view/259/277. Acesso em: 02 ago 2019.

STRARKEY, Chad. Recursos Terapêuticos em Fisioterapia. 4 ed. São Paulo: Manole, 2017.

VERSAGI, Charlotte Michael. Protocolos Terapêuticos de Massoterapia: técnicas passo a passo para diversas condições clínicas. Barueri, São Paulo: Manole, 2012.

WOOD, E. C; BECKER, P. D. Técnicas de Massagem de Beard. 5. ed. Rio de Janeiro: Elsevier, 2008.

Soumis : août 2019.

Approuvé : octobre 2019. 